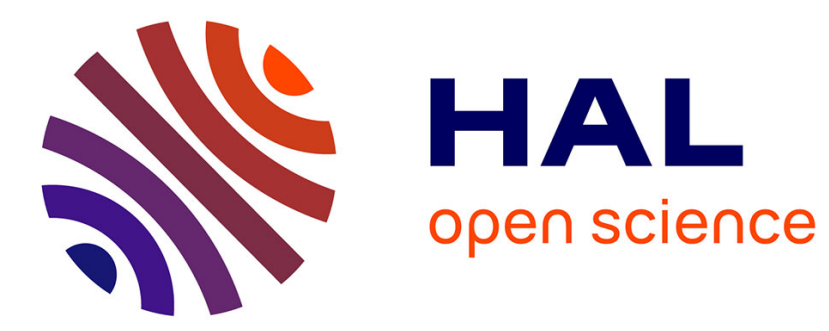

\title{
Le passé surcomposé et la valeur de parfait existentiel
} Denis Apothéloz

\section{To cite this version:}

Denis Apothéloz. Le passé surcomposé et la valeur de parfait existentiel. Journal of French Language Studies, 2010, 20 (2), pp.105-126. 10.1017/S0959269509990378 . hal-00867302

\section{HAL Id: hal-00867302 https://hal.science/hal-00867302}

Submitted on 29 Sep 2013

HAL is a multi-disciplinary open access archive for the deposit and dissemination of scientific research documents, whether they are published or not. The documents may come from teaching and research institutions in France or abroad, or from public or private research centers.
L'archive ouverte pluridisciplinaire HAL, est destinée au dépôt et à la diffusion de documents scientifiques de niveau recherche, publiés ou non, émanant des établissements d'enseignement et de recherche français ou étrangers, des laboratoires publics ou privés. 


\title{
Le passé surcomposé et la valeur de parfait existentiel ${ }^{1}$
}

\author{
DENIS APOTHÉLOZ
}

Université de Nancy 2, laboratoire ATILF (UMR 7118)

(Received June 2008; revised November 2008; first published online I2 November 2009)

\section{A B S T RAC T}

L'objectif de cet article est d'établir que dans l'un de ses emplois, traditionnellement qualifié de 'régional', le passé surcomposé est une forme verbale qui grammaticalise la valeur habituellement appelée 'parfait existentiel'. Dans une première partie, on dresse un panorama des principales propriétés du parfait existentiel, tel que l'exprime en français le passé composé, en distinguant propriétés sémantiques et propriétés discursives. On montre en particulier que cette valeur implique un prédicat existentiel lié à un intervalle de validation. Dans une seconde partie, on développe un certain nombre d'arguments établissant que le passé surcomposé grammaticalise cette valeur. On présente un tableau général des principaux emplois du surcomposé, et on analyse plusieurs exemples pour mettre au jour les points communs et les différences entre le passé composé et le passé surcomposé existentiels, notamment dans leur rapport avec les adverbiaux temporels quantifieurs de fréquence. On montre que le passé surcomposé existentiel est fréquemment utilisé pour établir un contraste entre le passé et le présent.

\section{INTRODUCTION}

En aspectologie anglaise, on appelle 'parfait existentiel' un emploi du present perfect dans lequel cette forme indique que la situation s'est produite une fois au moins dans le passé (McCawley, I97I). Cette valeur est appelée aussi 'passé indéfini' (Leech, I97I), 'parfait d'expérience' (Comrie, I976), parfois encore 'parfait incident' (Cohen, I989). Elle peut être illustrée par l'exemple suivant, emprunté à Comrie (I976: 54): I have (on some occasion in the past) got up at five o'clock, formulation qu'on pourrait traduire en français par Il m'est arrivé de me lever à cinq heures du matin. Leech (I97I) et Comrie (I976) signalent par ailleurs qu'il y a en anglais, pour traduire l'énoncé français Bill est allé en Amérique, deux formulations possibles. La première utilise le verbe 'to go' et produit un énoncé à valeur de présent résultatif (Bill has gone to America); elle implique que Bill est actuellement en Amérique, ou est en route pour l'Amérique (autrement dit elle implique l'absence hic et nunc de Bill). La

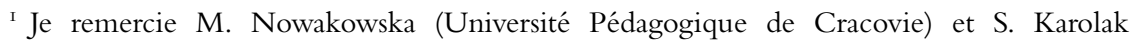
(Académie des Sciences de Pologne) qui ont lu diverses versions préparatoires de cet article et m'ont permis d'en améliorer très sensiblement la qualité. 
seconde utilise 'to be' et produit un énoncé à valeur de parfait existentiel (Bill has been to America); elle signale seulement que Bill, au cours de son existence passée, est allé une fois au moins en Amérique. C'est cette seconde valeur qui va nous intéresser tout au long de cet article. ${ }^{2}$

Il est frappant de constater que le parfait existentiel est rarement mentionné en aspectologie française (voir toutefois Vet, I992; Ritz, 2002; Desclés et Guentchéva, 2003), alors qu'il est abondamment discuté dans d'autres traditions linguistiques, anglaise et slave notamment. Quand cette valeur est évoquée, à propos du passé composé, c'est généralement assez rapidement et sans analyse véritablement approfondie. Nous allons donc, dans la première partie de cet article, présenter les principales propriétés du passé composé utilisé en parfait existentiel. Cet exposé, relativement long mais nécessaire, permettra ensuite d'aborder la question du passé surcomposé, en particulier dans l'une de ses variantes, désignée habituellement comme 'régionale'.

Comme le font beaucoup d'aspectologues, nous utiliserons le terme de 'situation' pour désigner le procès dénoté par la forme verbale ou prédicative étudiée, quelle que soit la nature de ce procès.

Nos exemples proviennent de trois sources: deux ouvrages de géographie des $\mathrm{XVIII}^{\mathrm{e}}$ et $\mathrm{XIX}^{\mathrm{e}}$ siècles, divers textes faisant partie de la base Frantext (marqués ' $F$ '), et des forums de discussion Internet. Pour des raisons méthodologiques évidentes, nous avons conservé l'orthographe d'origine dans tous les exemples. Cet avertissement vaut surtout pour les exemples issus de forums sur Internet et pour ceux des ouvrages de géographie. Les exemples non référencés ont été forgés pour les besoins de la démonstration ou sont des manipulations d'exemples référencés.

\section{PROPRIÉTÉS DU PARFAIT EXISTENTIEL}

La liste des propriétés présentée ci-dessous vise surtout à donner à notre démarche un caractère méthodique et n'a aucune prétention à l'exhaustivité. Nous distinguerons entre propriétés sémantiques et propriétés discursives. Nous n'examinerons ici que le cas du passé composé, mais il existe également des emplois existentiels du plus-que-parfait et du futur antérieur (Apothéloz, 2009).

\subsection{Propriétés sémantiques}

(i) Comrie (I976: 58) donne la définition suivante du parfait existentiel: 'The experiential perfect indicates that a given situation has held at least once during

2 Il y a en français une variation similaire (il est allé il a été), où 'être' est utilisé comme une sorte d'allomorphe de 'aller'. Il est possible que cette variation, dont la deuxième forme est régulièrement stigmatisée par le discours normatif, soit simplement la trace d'un processus de grammaticalisation identique à ce qu'on observe en anglais, mais jamais arrivé à son terme. 
some time in the past leading up to the present'. Cette définition comporte un élément supplémentaire, relativement à ce que nous avons écrit en introduction: 'during some time in the past leading up to the present'. Autrement dit, l'intervalle temporel à l'intérieur duquel la situation est dite s'être produite s'étend jusqu'au moment de l'énonciation. Comme l'a noté McCawley (I97I, I98 I), cela implique qu'un type de situation qui, pour une raison ou une autre, n'est pas concevable comme reproductible, ne peut pas être désigné au moyen d'un parfait existentiel. Tel serait le cas des exemples ci-dessous.

(I) John L. Austin est né à Lancaster.

J'ai fait la connaissance de Georges au Japon.

Il a fait sa première communion en 1980.

Dans ces exemples, il est chaque fois question d'une situation dont la caractéristique principale est qu'elle ne peut pas (ou ne peut que très difficilement) être répétée: en principe on ne nait, on ne fait la connaissance de quelqu'un, on ne fait sa première communion, qu'une seule fois. Or l'une des caractéristiques du parfait existentiel est justement de désigner une situation qui doit pouvoir être répétée, et cela dans une période qui s'étend jusqu'au présent de l'énonciation. Pour cette raison, il est pratiquement impossible de trouver un contexte où les énoncés (I) puissent être interprétés comme des parfaits existentiels.

Il est intéressant de noter que cette répétabilité implique, de fait, une dissociation entre instance de situation et type de situation. À strictement parler, une situation est toujours unique et ne saurait se répéter. Par conséquent, tout constat ou toute indication de répétabilité suppose nécessairement que la situation désignée soit considérée comme ressortissant à un type.

(ii) À cette possibilité d'une répétition, s'ajoute le fait que la situation désignée n'est pas localisée précisément dans le temps. Tel est clairement le cas dans les exemples (2)-(3).

(2) Souvenez-vous, Claire, que vous avez jadis repoussé mes conseils avec un mépris immérité et qu'aujourd'hui vous le regrettez inutilement. (A. Maurois, Ariel ou la vie de Shelley, F)

(3) [...] qu'est-ce que tu veux, moi j'ai changé et toi tu es resté le même. Cela a beau te gêner, j'ai été à la guerre. J'ai vu les pires choses et elles étaient simples, calmes, saines, - oui, saines même dans la férocité et dans la mort. J'ai vu des hommes qui agonisaient sur ma poitrine et qui ne se plaignaient pas. J'ai vu des hommes qui donnaient leur vie l'un pour l'autre [...] (H. de Montherlant, L'Exil, F)

Cette absence de localisation temporelle précise est souvent donnée comme l'une des caractéristiques principales du parfait existentiel.

(iii) Le parfait existentiel suppose en revanche toujours un intervalle de validation'. Tel est le nom que nous donnerons à l'intervalle temporel à l'intérieur duquel l'assertion d'existence est valide. Cet intervalle peut correspondre à des informations implicites, glosables par des expressions telles que au cours de mon (ton, son, etc.) existence, dans le passé, ou jusqu'à aujourd'hui. Pour le passé composé à 
valeur de parfait existentiel, il s'étend, à défaut de spécification, jusqu'au moment de l'énonciation.

(iv) Le nombre d'occurrences de la situation et l'étendue de l'intervalle de validation peuvent être spécifiés au moyen d'adverbiaux ou de compléments. C'est ce qui se passe dans (4) et (5) pour le nombre d'occurrences de la situation.

(4) J'ai plusieurs fois tiré sur des agents de police (au sens de: 'plusieurs fois, dans le passé, il m'est arrivé de tirer sur des agents de police') (in: Karolak, 2007: 50)

(5) D'ailleurs, aux yeux des maoris, rien de plus naturel que de se manger les uns les autres. Les missionnaires les ont souvent interrogés à propos du cannibalisme. Ils leur ont demandé pourquoi ils dévoraient leurs frères; à quoi les chefs répondaient que les poissons mangent les poissons [...] (J. Verne, Les enfants du capitaine Grant)

L'extrait (5) comporte deux passés composés successifs à valeur de parfait existentiel. Le premier est accompagné de l'adverbe souvent, qui contribue d'ailleurs à sélectionner cette interprétation. Le second n'est accompagné d'aucun adverbial, mais il est lui aussi sous la portée sémantique de souvent. Le texte continue ensuite avec un imparfait (répondaient), ce qui confirme et proroge l'itérativité potentielle et l'indéfinitude du parfait existentiel. Il est intéressant de noter que si ces passés composés étaient interprétés comme des prétérits, le texte continuerait avec un passé composé (ont répondu) également à valeur prétéritale. Ce que montre la comparaison de $(5)$ et de (6):

(6) Les missionnaires les ont interrogés à propos du cannibalisme. Ils leur ont demandé pourquoi ils dévoraient leurs frères; à quoi les chefs ont répondu que les poissons mangent les poissons.

Quant à l'étendue de l'intervalle de validation, elle peut également être spécifiée, ce qui revient pratiquement à en diminuer l'extension temporelle, comme dans (7).

(7) Pendant trois quatre ans son absence m'a souvent paru insupportable. (E. Hanska, J'arrête pas de t'aimer, F)

(v) Le parfait existentiel véhicule, comme son nom l'indique, une prédication d'existence: le contenu propositionnel $p$ de l'énoncé y est dominé par une signification qu'on peut gloser ainsi: il est arrivé que $\mathrm{p}$, il s'est passé $\mathrm{p}, \mathrm{p}$ a eu lieu. Nous appellerons 'prédicat existentiel implicite' ce composant 'il est arrivé que', en général non formulé, des énoncés à valeur de parfait existentiel. ${ }^{3}$ En termes plus techniques, cela signifie que le contenu propositionnel d'un énoncé véhiculant la valeur de parfait existentiel est lié par un quantificateur existentiel (que traduit le prédicat existentiel implicite), dans l'espace défini par l'intervalle de validation. Vet (I992:

${ }^{3}$ Le parfait existentiel produit donc ce que Jespersen (I924) appelait des 'phrases existentielles', plus exactement un type particulier de phrases existentielles. Comme toute tentative de glose, celle que nous proposons pour restituer le sens de ce type de phrase existentielle peut prêter le flan à la critique. Elle présente toutefois l'avantage d'être compatible aussi bien avec des situations non intentionnelles (il est arrivé qu'il perde connaissance) qu'intentionnelles (il est arrivé que nous sortions à deux heures du matin). 
49) appelle cet intervalle 'domaine de quantification'. Tous les exemples donnés ci-dessus pourraient d'ailleurs être reformulés au moyen du tour impersonnel il est arrivé que, qui est un exposant du composant existentiel. Montrons-le sur (2)-(5):

$\left(2^{\prime}\right)$ Souvenez-vous, Claire, qu'il vous est jadis arrivé de repousser mes conseils avec un mépris immérité...

$\left(3^{\prime}\right) \quad i l$ m'est arrivé d'aller à la guerre. Il m'est arrivé de voir les pires choses... Il m'est arrivé de voir des hommes qui agonisaient sur ma poitrine...

$\left(4^{\prime}\right)$ Il m'est arrivé plusieurs fois de tirer sur des agents de police.

$\left(5^{\prime}\right)$ Il est souvent arrivé que les missionnaires les interrogent à propos du cannibalisme...

Ces formulations montrent également que les adverbiaux de (3) et (4) portent sur le prédicat existentiel implicite, autrement dit sur l'affirmation du concept d'existence.

(vi) De nombreux auteurs ont noté que le parfait existentiel est fréquemment en co-occurrence avec certains adverbiaux temporels (e.g. Leech, I97I; Dahl, I985; Guentchéva, I990; Karolak, 2007). Ces adverbiaux appartiennent principalement à deux catégories: d'une part, des quantifieurs de fréquence (déjà, un jour, une fois, rarement, parfois, plus d'une fois, souvent, toujours, jamais, pas une seule fois); d'autre part des expressions localisant le procès dans un passé plus ou moins lointain et indéterminé (autrefois, jadis, il y a longtemps). Toutes ces expressions fonctionnent, avec le passé composé, comme des indicateurs voire comme des déclencheurs de l'interprétation existentielle. ${ }^{4}$ Voici quelques exemples où l'un de ces adverbiaux apparait:

(8) Je refuse de nourrir mon python de souris vivantes, voilà, lui dis-je. C'est inhumain. Et il refuse de bouffer autre chose. Avez-vous déjà vu une pauvre petite souris face à un python qui va l'avaler? C'est atroce. (E. Ajar, Gros-Câlin, F)

(9) Beaucoup d'artistes célèbres ont eu des manies bizarres aux heures du travail. Balzac s'en attribuait plus qu'il n'en avait réellement [...]. Je l'ai surpris plus d'une fois, en plein jour, travaillant comme tout le monde, sans excitants, sans costume, sans aucun signe d'enfantement douloureux, riant dès l'abord, l'œil limpide et le teint fleuri. (G. Sand, Histoire de ma vie, F)

(Iо) As-tu jamais vu un petit poisson qui essaie de remonter un courant rapide, et que la force du courant maintient à la même place, immobile et frémissant. (H. de Montherlant, Pasiphaé, F)

(I I) [...] tandis que les philosophes de l'Antiquité ont parfois pressenti la véritable nature des fossiles, pendant tout le moyen âge et la Renaissance on a fait à leur sujet les suppositions les plus curieuses. (M. Boule, Conférences de géologie, F)

${ }^{4}$ Leech (I97I: 556 ) note que le present perfect est rarement utilisé comme parfait existentiel sans être accompagné d'un adverbe comme ever, never ou before, qui fonctionne comme une sorte de 'renforcement' de la valeur existentielle. 


\section{Denis Apothéloz}

L'exemple (9) fait bien voir le lien qu'il y a entre l'interprétation existentielle et la présence d'un tel adverbial. Si on y supprime plus d'une fois, la lecture existentielle n'est plus du tout assurée. Du moins, elle entre en concurrence avec une lecture prétéritale du passé composé (qui serait alors traduit par un simple past en anglais). C'est un point sur lequel nous reviendrons.

(vii) Le parfait existentiel a également des affinités avec la détermination indéfinie du syntagme nominal (Karolak, 2007). Ce point concerne l'expression désignant le second actant, comme le montre la comparaison des deux formulations ci-dessous.

(I2) Avez-vous déjà vu un cadavre?

Avez-vous déjà vu le cadavre?

La détermination indéfinie peut donc contribuer à sélectionner l'interprétation existentielle du passé composé, de la même manière que les adverbiaux temporels mentionnés plus haut.

(viii) Les aspectologues soulignent souvent que le parfait existentiel vise à signifier l'actualité d'une certaine expérience acquise, les traces psychologiques laissées par la situation désignée (d'où l'autre appellation: 'parfait d'expérience'). Ce fait apparait clairement dans certains exemples discutés plus haut, en particulier dans (3): j'ai été à la guerre... J'ai vu des hommes qui agonisaient. . ; dans (4): J'ai plusieurs fois tiré sur des agents de police; dans (8): Avez-vous déjà vu une pauvre petite souris. . ; dans (9): Je l'ai surpris plus d'une fois, en plein jour. ..; dans (Io): As-tu jamais vu un petit poisson qui essaie de...; ainsi que dans (I2). Le type d'énoncé existentiel examiné ici vise non seulement à asserter ou à nier l'existence d'une situation, mais aussi à indiquer que cette situation a des effets dans le présent de l'énonciation: c'est dans l'actualité de l'énonciation que l'assertion d'existence trouve sa pertinence. En cela, il s'agit bien d'une valeur de parfait, signifiant une certaine 'résultativité'.

\subsection{Propriétés discursives}

Les caractéristiques qui viennent d'être énumérées ont diverses incidences sur les emplois discursifs qui peuvent être faits des énoncés comportant un parfait existentiel. Examinons quelques-unes de ces incidences.

5 Nous utilisons ici le terme de 'résultativité' dans un sens plus général qu'on ne le fait habituellement. Beaucoup de linguistes (e.g. Comrie, 1976; Guentchéva, I990) distinguent, parmi les diverses valeurs de parfait, le parfait existentiel et le parfait résultatif. Pour eux, le parfait existentiel n'est donc pas résultatif. Dans la terminologie utilisée ici, le terme de 'parfait' est d'abord sémantique, et désigne la propriété de certains tiroirs consistant à exprimer un certain rapport entre la situation dénotée par le verbe et un moment de référence postérieur à cette situation. Dans cette perspective, tout parfait est par définition résultatif. Le parfait habituellement qualifié d"existentiel' ou d"expérience', dans la mesure où il exprime le rapport temporel ci-dessus, appartient donc de plein droit à la famille des parfaits. Dans Apothéloz (2009.) et dans Apothéloz et Nowakowska (à par.), nous distinguons résultativité 'sémantique' et résultativité 'pragmatique', et montrons que la résultativité du parfait existentiel est de type pragmatique. 
(ix) Les énoncés comportant un parfait existentiel présentent une organisation informationnelle particulière. En effet, dans la mesure où leur prédicat principal est le prédicat existentiel lui-même (celui qu'explicite le tour impersonnel il est arrivé $q u e)$, ces énoncés sont entièrement rhématiques. ${ }^{6}$ Cependant cela ne signifie pas, comme on l'écrit parfois, que tout soit nécessairement 'nouveau' dans ces énoncés. Une anaphore pronominale, par exemple, n'y est nullement exclue.

Cette organisation informationnelle particulière a diverses conséquences sur les contextes où ces énoncés peuvent apparaître. Prenons l'exemple (4): J'ai plusieurs fois tiré sur des agents de police. Interprété comme parfait existentiel, cet énoncé pourrait être utilisé pour répondre à des questions comme: Qu'est-ce qui me prouve que vous n'hésiteriez pas à utiliser une arme?, Avez-vous déjà eu des altercations avec les forces de l'ordre?, etc. Mais il ne pourrait pas être utilisé pour répondre à la question: Qu'avez-vous fait alors? En réponse à cette question, l'énoncé aurait nécessairement une interprétation non-existentielle, c'est-à-dire que le passé composé y aurait une valeur de prétérit. Prenons encore l'exemple suivant, qui se trouve à la fin de l'extrait (3): J'ai vu des hommes qui donnaient leur vie l'un pour l'autre. Une réaction appropriée à cet énoncé, s'il est interprété comme parfait existentiel, pourrait être la question: Combien de fois cela vous est-il arrivé? Mais cette même question serait inappropriée si on interprétait le passé composé de cet extrait comme un prétérit (cf. Michaelis, I998: 224).

(x) Compte tenu de leurs propriétés temporelles, les énoncés au parfait existentiel se prêtent à des énumérations non chronologiques, c'est-à-dire dans lesquelles la chronologie des situations successivement rapportées est indifférente. L'extrait (I 3) en est une illustration.

(І3) [...] je n'ai pas toujours eu ce bras infirme... j’ai été jeune, ardent... j’ai aimé... j’ai désiré... je l'ai laissé voir... et jamais, jamais, vous entendez bien, pas une seule fois dans toute mon existence, je n'ai vu un regard un peu chaud répondre tout à coup à mon regard. . jamais je n'ai senti un corps frissonner près du mien... un désir, enfin (R. Martin du Gard, Un taciturne, F)

(xi) Les énoncés au parfait existentiel ont également certaines affinités avec l'argumentation. Il s'agit d'un point qui mériterait à lui seul une étude détaillée, mais que nous ne pouvons aborder ici que succinctement. Le problème est le suivant. On peut considérer que la dimension argumentative du discours se manifeste notamment par un type particulier d'enchaînement, dans lequel un énoncé (une 'raison') vise à étayer un autre énoncé (une 'conclusion'), le tout formant un 'argument' (sur ces notions, voir Apothéloz et al., I993). Précisons que la conclusion n'est pas toujours explicitement formulée, et qu'elle peut demeurer un implicite de la communication. On observe alors que les énoncés au parfait existentiel fonctionnent souvent comme des raisons données en faveur d'une

${ }^{6}$ Sur cette question, voir Kuroda (I973), Lambrecht (I994), Kęsik (I998), Kleiber (200I), Cornish (2005). 
conclusion. Le contenu de cette dernière peut consister dans les prolongements psychologiques actuels de la situation désignée, en un mot dans l'"expérience'. Ce rapport argumentatif de raison à conclusion apparaît clairement dans plusieurs de nos exemples, notamment dans (9), répété ci-dessous.

(9) Beaucoup d'artistes célèbres ont eu des manies bizarres aux heures du travail. Balzac s'en attribuait plus qu'il n'en avait réellement [...]. Je l'ai surpris plus d'une fois, en plein jour, travaillant comme tout le monde, sans excitants, sans costume, sans aucun signe d'enfantement douloureux, riant dès l'abord, l'oil limpide et le teint fleuri. (G. Sand, Histoire de ma vie, F)

G. Sand veut montrer ici que selon elle, les manies attribuées à Balzac étaient en partie de la mystification (cf. Balzac s'en attribuait plus qu'il n'en avait réellement). Cette 'conclusion' est étayée par un témoignage, qui est l'énoncé au parfait existentiel. Ce rapport argumentatif pourrait d'ailleurs fort bien être souligné formellement (cf. en effet, je l'ai surpris plus d'une fois...). On pourrait analyser de façon analogue (3), (8), (го), (г 3 ).

\section{LE PASSÉ SURCOMPOSÉ}

Compte tenu de l'objectif de cet article, nous n'aborderons que de façon très sommaire la question de la morphologie du passé surcomposé, de même que celle de sa géographie linguistique. Pour la même raison, nous laisserons pratiquement de côté toute considération diachronique, générationnelle ou sociolinguistique. Sur ces différents points, nous renvoyons le lecteur aux travaux de Cornu (I953), Dauzat (I954), Stefanini (I954), Walter (I98 I, I988), Hill (I984), Jolivet (I984), Engel (I996), Wilmet (I997), Carruthers (I994, I998, I999), Schaden (2007) en particulier. On trouvera également dans Ayres-Bennett et Carruthers (I992) une revue très complète du traitement dont les formes surcomposées ont fait l'objet dans les grammaires, entre le $\mathrm{XVI}^{\mathrm{e}}$ et le $\mathrm{XX}^{\mathrm{e}}$ siècles De même, nous ne parlerons pas des autres tiroirs surcomposés: plus-que-parfait surcomposé, futur antérieur surcomposé, conditionnel surcomposé, etc.

\subsection{Morphologie}

Comme son nom l'indique, le passé surcomposé est un tiroir verbal doublement composé, que Tesnière (I939) décrivait comme comportant un auxiliaire lui-même composé, suivi du participe passé du verbe lexical. Il en résulte des formes comme: je l'ai eu mangé ou il a été parti. Cependant cette description est loin d'être satisfaisante. D'une part, en effet, elle ne rend pas compte des formes surcomposées des verbes pronominaux, dont le participe passé est toujours précédé de $e u$, lui-même précédé d'une forme fléchie de l'auxiliaire 'être':

(I4) il s'est eu lavé

je me suis eu assis 
D'autre part, on observe pour les autres verbes normalement conjugués avec 'être', une certaine variation dans la formulation du passé surcomposé, le type il a été parti cédant parfois la place au type il est eu parti, sur le modèle des verbes pronominaux. Dans certaines régions de la francophonie, en particulier dans le domaine franco-provençal, seule la deuxième de ces formes est pratiquement utilisée, comme si la formation du passé surcomposé des verbes pronominaux était généralisée à tous les verbes à auxiliaire 'être'. D'où des formes comme les suivantes, tout à fait régulières dans les régions en question:

(I 5) il est eu tombé

on est eu rentré très tard

Toutes ces formes mettent en cause l'analyse consistant à considérer que le passé surcomposé est construit à partir du temps composé de l'auxiliaire (voir aussi Wilmet, I997: 337-338).

Quoi qu'il en soit, la coexistence même de formes comme il a été parti et il est eu parti conduit à se demander si on est en présence d'un fait de pure variation grammaticale, ou de deux tiroirs distincts (quelle que soit par ailleurs leur stabilité, leur degré d'intégration dans le système, l'extension géographique de leur emploi, etc.). ${ }^{7}$ A notre connaissance, cette question est rarement abordée dans la littérature.

\subsection{Contextes grammaticaux et répartition géographique}

Le passé surcomposé apparait principalement dans trois contextes grammaticaux. Toutefois ces emplois ne peuvent pas être tous considérés comme faisant partie du français 'standard'. Si l'on s'en tient à l'aire européenne, la situation est approximativement la suivante.

Le premier contexte grammatical est celui formé par les subordonnées temporelles en après que, dès que, aussitôt que, quand, lorsque, une fois que, à peine. . que, etc. Cet emploi est généralement considéré comme standard, c'est-à-dire non limité à une aire géographique particulière. Il semble spécialement fréquent avec les verbes désignant la phase terminale d'une situation (finir, achever, terminer).

(i6) Dès que j'ai eu dit que mon frère était dans les ordres, l'atmosphère changea, et je me vis réintégré dans le sein de l'Église. (R. Vrigny, La nuit de Mougin, F)

( I7) [...] aussitôt que Monsieur l'a eu quitté [...], M. Bonacieux a pris son chapeau, a fermé sa porte et s'est mis à courir par la rue opposée. (A. Dumas, Les trois mousquetaires)

7 Pour autant, d'ailleurs, que la forme il a été parti soit un surcomposé, étant donné l'ambivalence que peuvent présenter les verbes auxiliés par 'être': séquence 'copule + attribut', ou tiroir verbal. On sait que le passé composé peut parfois être confondu avec une construction avec attribut de l'objet (cf. les deux interprétations de il l'a cassé). Il en va de même du passé surcomposé. Voir cet exemple: Et ce soir, il a amené dans le salon de la princesse [...] un petit bonhomme pincé, prétentieux, parlant du nez, d'un vilain nez, qu'il a eu cassé à l'accident de chemin de fer de la rive gauche (Journal des Goncourt, F). 


\section{Denis Apothéloz}

(I 8) Après que j'ai eu parlé, un Anglais s'est approché de M. Talbot [...]. (V. Hugo, Correspondance, F)

(i9) Quand elle a eu terminé la chanson, on a applaudi. (L. Malet, Le soleil n'est pas pour nous, F)

Dans cet environnement, le passé surcomposé sert à établir une corrélation temporelle entre deux situations, et est presque toujours conjoint avec un passé composé (à cet égard, (I6) fait plutôt figure d'exception). Grammairiens et linguistes ont noté depuis longtemps que dans ce contexte, le surcomposé joue le même rôle, vis-à-vis du passé composé, que le passé antérieur vis-à-vis du passé simple. ${ }^{8}$ Au plan aspectuel, il signifie le plus souvent la résultativité dans le passé, mais parfois aussi la processualité dans le 'passé du passé' (sur l'opposition 'processuel' vs 'résultatif, voir Confais (I995)). Beauzée (I765), qui ne décrit dans son article que les emplois en subordonnée temporelle (où il y a 'comparaison' de la temporalité de deux situations), qualifiait ce surcomposé de 'prétérit indéfini comparatif.

Il est intéressant d'examiner comment se combinent ici les potentialités aspectuelles du surcomposé et le sens de la conjonction.

Les conjonctions dès que et aussitôt que impliquent une durée et pointent le début de cette durée. Combinées avec le passé surcomposé, elles mettent en évidence l'état résultatif et induisent donc une interprétation résultative du surcomposé. En utilisant les trois 'points' de Reichenbach (1947), on peut décrire ainsi cette configuration aspectuo-temporelle: le point de référence $(\mathrm{R})$ est postérieur au point de l'événement (E), et le couple E-R est lui-même antérieure au point de la parole (S). Dans (I6), la conjonction pointe sur le moment à partir duquel l'information 'mon frère est dans les ordres' a été communiquée, de sorte que la signification de la subordonnée équivaut pratiquement à la paraphrase 'à partir $d u$ moment où il fut connu que mon frère était dans les ordres'. Dans (I 7), aussitôt que Monsieur l'a eu quitté pointe sur le moment à partir duquel l'individu nommé 'Monsieur' n'est plus aux côtés de Bonacieux. Il en va de même avec la conjonction quand dans (I9): on pourrait d'ailleurs lui substituer dès que ou aussitôt que. Comme l'a bien vu Stefanini (I954), un passé composé à la place du surcomposé serait souvent aspectuellement problématique dans ce contexte. En effet, d'une part le passé composé de la proposition principale, qui a ici valeur de prétérit, contraint d'interpréter celui de la subordonnée temporelle comme un temps du passé; mais d'autre part, le passé composé à valeur de passé est en principe toujours une forme processuelle et non pas résultative (il ne peut être résultatif que dans le présent). Or

${ }^{8}$ Notamment les grammairiens des XVII ${ }^{\mathrm{e}}$ et XVIII ${ }^{\mathrm{e}}$ siècles, mais aussi Clédat (I926), Frei (I929), Gougenheim (I939), Tesnière (I939), Cornu (I953), Benveniste (I959), Martinet (I979), Laca (2005), pour n'en citer que quelques-uns. Leur raisonnement est généralement le suivant: compte tenu de la propension du passé composé à être utilisé comme un prétérit et à empiéter ainsi sur le passé simple, le besoin se fait sentir de remplacer, dans le tandem passé antérieur-passé simple, le passé antérieur par une forme surcomposée. Quand il eut terminé, il sortit devient ainsi quand il a eu terminé, il est sorti, qui n'est autre que la transposition dans le passé de quand il a terminé, il sort. 
ici, la conjonction force en quelque sorte cette valeur résultative. C'est sans doute dans ce type de contexte que la nécessité du passé surcomposé se fait le plus sentir. ${ }^{9}$

Dans (i 8), en revanche, la forme surcomposée est processuelle. Cette différence d'avec les exemples précédents tient au sens même de la conjonction après que et à la manière dont elle se combine avec la forme verbale. La forme verbale qui suit après que désigne nécessairement la phase processuelle de la situation, et cela jusqu'à sa borne terminale. Dans (I8) (après que j'ai eu parlé...), il s'agit du temps que dure la prise de parole. Certes, la subordonnée désigne ici la phase qui suit la phase processuelle: après que j'ai eu parlé désigne le moment à partir duquel la prise de parole est terminée. Mais compte tenu du sens de après que, la forme verbale j'ai eu parlé de cette proposition désigne, elle, le moment même de la prise de parole. Le point $\mathrm{R}$ est donc ici concomitant du point E. Etant donné que le moment de cette parole est antérieur à celui de la situation désignée par la proposition principale (un Anglais s'est approché de M. Talbot), il s'agit de processualité dans le 'passé du passé'. ${ }^{10}$

Le deuxième contexte grammatical est celui formé par les propositions indépendantes ou principales accompagnées d'un adverbial temporel comme rapidement, bientôt, vite, plutôt, ou un syntagme prépositionnel équivalent, i.e. indiquant une certaine 'précipitation' (Barceló et Bres, 2006). Cet emploi est généralement considéré comme faisant partie lui aussi du français standard.

(20) Et pourtant, on a eu fait très rapidement, là encore, le tour de la question. L'inventaire. Tous les bustes, [...] les moulures [...], on les a eu bientôt, sans exception, tous recensés. (Bayon, Le Lycéen, F)

(2 I) Les Belges, on a eu vite saisi qu'il y en a deux sortes: les Wallons et les Flamands. (F. Cavanna, Les Russkoffs, F)

(22) En cinq minutes, il a eu disparu. (H. Alain-Fournier, Correspondance avec J. Rivière, F)

Le passé surcomposé exprime, dans ces exemples, la résultativité dans le passé. Dans (20) par exemple, on a eu fait très rapidement [...] le tour de la question réfere temporellement au moment à partir duquel le tour de la question a été fait, et indique que ce moment a été atteint rapidement. Dans ce même extrait, on les a

${ }^{9}$ Voir aussi Imbs (I960: I02). Le type du prédicat verbal joue aussi un rôle, ici. Pour les verbes transitionnels (au sens de Vet, I980) instantanés, l'écart temporel entre la phase transitionnelle proprement dite et la phase résultative est minime. Pour cette raison, probablement, le passé composé dans la subordonnée temporelle passe relativement inaperçu, en dépit du problème mentionné ci-dessus (cf. Quand il a fini son repas, il s'est mis au travail). Mais avec d'autres types de prédicats, duratifs transitionnels ou nontransitionnels (faire ses courses, attendre), la nécessité du passé surcomposé se fait davantage sentir: des formulations comme Quand il a fait ses courses, il s'est mis au travail, ou Quand il a attendu une demi-heure, il est allé se coucher, sont ressenties comme extrêmement problématiques.

10 A noter que la conjonction quand est, elle, sous-spécifiée relativement à cette distinction: la forme il a mangé est processuelle dans quand il a mangé, il pleuvait, mais résultative dans quand il a mangé, il sort. Dans le premier cas, la conjonction met en évidence le temps du procès proprement dit; dans le second, son résultat. 


\section{Denis Apothéloz}

eu bientôt [...] tous recensés désigne le moment à partir duquel le recensement est terminé. Dans ces trois exemples, $\mathrm{R}$ est donc postérieur à $\mathrm{E}$.

Le troisième contexte grammatical n'a rien à voir avec la notion de corrélation temporelle. C'est pourquoi cet emploi a été qualifié d'absolu (Cornu, I953). Géographiquement, il est limité aux domaines d'oc et franco-provençal, Suisse romande comprise, où il paraît jouir d'une faveur particulière (Cornu, I953; Walter, I988; Carruthers, I994, I998).

(23) Comme M. le curé nous l'a eu fait ressortir bien des fois, s'il y a une femme qui peut compter aller près du bon Dieu, sûr et certain que c'est elle. (M. Proust, Du côté de Guermantes, F)

(24) Les habitans, aux mœurs simples et superstitieuses, racontent beaucoup d'histoires sur un vieux nain qu'on a eu vu sur leurs pâturages. (M. Lutz, Dict. géogr. et stat. de la Suisse, trad. de l'allem., Lausanne, I836)

(25) Je rentre de la chasse aux œufs avec mon fiston et le chien. Après 2 h3o de marche dans le village, on rentre crevés mais heureux. On a réalisé un beau score: Io œufs. Il faut savoir qu'on est eu rentré avec un seul ceuf... (http://forum.aufeminin.com/forum/)

Faute de lui trouver une caractérisation aspectuo-temporelle satisfaisante, on qualifie souvent cet emploi de 'régional', par opposition au surcomposé 'général' des deux contextes précédents (Foulet, I925; Jolivet, I986; Carruthers, I994) ${ }^{11}$. Cornu (I953) et Nilsson-Ehle (I953) datent les premières attestations de cet emploi du XIV ${ }^{\mathrm{e}}$ siecle.

Beaucoup de choses ont été écrites sur les aspects sociolinguistiques de cet emploi, en particulier ses rapports avec la norme, qui ont parfois donné lieu à controverses. Le fait qu'il soit, semble-il, davantage stigmatisé que les deux autres (Carruthers, I998) n'est peut-être pas sans rapport avec la difficulté que les linguistes ont toujours éprouvée à en analyser la valeur aspectuo-temporelle.

Quoi qu'il en soit, un exemple comme (24) atteste qu'à une époque relativement récente, dans l'aire géographique indiquée plus haut, ce type de surcomposé pouvait se rencontrer dans les écrits les plus normés (l'exemple de Proust est du discours rapporté direct). Voici deux autres exemples extraits d'un ouvrage de géographie un peu antérieur au précédent.

(26) Cette mine a eu produit jusqu'à 24 mille marcs d'argent fin, dans une année; mais elle a beaucoup diminué. (H. Descombes, Géogr. universelle, Lausanne, I790. Un marc est une ancienne unité de poids.)

(27) Les habitans vivent longtemp, \& sont peu sujets aux maladies. Salmon dit qu'on y a eu levé jusqu'à dix mille hommes de troupes; ce qui prouveroit qu'il y a $\mathrm{eu}$, autrefois, une beaucoup plus grande population qu'aujourd'hui. (ibid.)

Nous avons trouvé I 3 exemples de ce type de surcomposé dans cet ouvrage. Il est intéressant de noter que le nom de son auteur est assez répandu en zone

11 Dauzat (I954) l'a qualifié de 'provençal', appellation évidemment impropre au plan de la géographie linguistique. Schaden (2007) parle quant à lui de 'superparfait'. 
franco-provençale. Toutes ces formes pourraient être produites aujourd'hui encore dans cette même zone géographique. Tel est le sentiment du signataire du présent article, qui est originaire de cette région.

S'il est vrai que ce type de surcomposé est aujourd'hui complètement absent dans les écrits normés, il n'en demeure pas moins que dans l'oral conversationnel ordinaire, il n'est pas véritablement rare.

Mais ce qui nous intéresse ici est son analyse sémantique. Or, à considérer (23)(27), on ne peut qu'être frappé par le fait que ces exemples présentent toutes les caractéristiques du parfait existentiel, tel que nous l'avons défini: nombre indéterminé d'occurrences de la situation désignée (avec ce que cela implique d'itérativité potentielle), absence de repérage temporel précis, prédicat existentiel implicite dont le contenu peut être paraphrasé par il est arrivé que, et exerçant son effet dans un intervalle de validation, etc. ${ }^{12}$ Ainsi $\left(23^{\prime}\right)-\left(27^{\prime}\right)$ semblent être des gloses exactes du sens de (23)-(27):

(23') Comme il est arrivé bien des fois à $M$. le curé de nous le faire ressortir,...

$\left(24^{\prime}\right)$ Les habitants [...] racontent beaucoup d'histoires sur un vieux nain qu'il est arrivé qu'on voie sur leurs pâturages.

$\left(25^{\prime}\right)$ Il faut savoir qu'il nous est arrivé de rentrer avec un seul œuf.

$\left(26^{\prime}\right)$ Il est arrivé que cette mine produise jusqu'à 24 mille marcs d'argent fin, dans une année.

$\left(27^{\prime}\right)$ Salmon dit qu'il est arrivé qu'on y lève jusqu'à dix mille hommes de troupes.

Aspectuellement, ces surcomposés sont des formes à la fois processuelles et résultatives: d'une part, elles désignent une occurrence passée (une au moins) d'un type de situation; mais d'autre part, elles désignent également une conséquence actuelle (expérience, trace mémorielle, etc.) de cette situation passée. C'est là aussi une caractéristique du parfait existentiel. Il en résulte que cet emploi du passé surcomposé se laisse difficilement décrire au moyen du système des trois points de Reichenbach (voir toutefois les suggestions de Kiparsky, s.d.).

Il est intéressant de noter que dans ces exemples, le passé surcomposé se trouve dans l'entourage de présents. Dans (23), on observe également la présence d'un quantifieur de fréquence (bien des fois).

Dans (25) et (27), on retrouve une autre propriété du parfait existentiel: sa valeur argumentative: dans (25), l'énoncé on est eu rentré avec un seul œuf sert à justifier le jugement suivant lequel une récolte de dix oufs est un 'beau score'; dans (27), on y a eu levé jusqu'à dix mille hommes permet d'étayer l'hypothèse selon laquelle il y a $e u$, autrefois, une beaucoup plus grande population qu'aujourd'hui.

12 L'observation selon laquelle l'emploi de ce surcomposé est assez fidèlement paraphrasé par le tour impersonnel 'il est arrivé que' a été faite il y a longtemps déjà. Voir par ex. Foulet (I925), Frei (I929), Brun (I93 I) cité par Géa (I995), Cornu (I953), Guiraud I965). Nous avons noté que, dans ses entretiens dirigés, Carruthers (I999: I9) utilise, comme 'elicitation technique', des questions du type: est-ce qu'il $y$ a des choses qu'il vous est arrivé de faire dans l'armée que vous ne referiez jamais?, vous avez déjà voyagé à l'étranger?, qui sont typiquement des énoncés au parfait existentiel. 
Les surcomposés de (23)-(27) sont donc, sur le plan sémantique, radicalement différents de ceux qui apparaissent dans les deux autres contextes. Ils ne pourraient par exemple pas figurer dans une subordonnée temporelle en quand, dès que, etc., ce qui est prévisible puisque le parfait existentiel est incompatible avec une localisation temporelle spécifique.

Nous voilà donc en présence d'un autre tiroir verbal prédisposé à exprimer la valeur de parfait existentiel. ${ }^{13}$ C'est cet emploi que nous allons maintenant analyser plus en détail. Nous appellerons désormais les surcomposés de ce type 'surcomposéE'.

\section{SURComposé-E et passé Composé a VAleur de parfait EXISTENTIEL}

Nous voudrions maintenant développer quelques arguments montrant que le surcomposé-E est une forme qui grammaticalise, au sens fort du terme, la valeur de parfait existentiel. Pour ce faire, nous allons comparer des exemples de surcomposésE et de passés composés à lecture existentielle.

Reprenons (23)-(27) et transposons-les au passé composé.

(23a) Comme M. le curé nous l'a fait ressortir bien des fois...

(24a) Les habitants [...] racontent beaucoup d'histoires sur un vieux nain qu'on a vu sur leurs pâturages.

(25a) On a réalisé un beau score: Io œufs. Il faut savoir qu'on est rentré avec un seul œuf.

(26a) Cette mine a produit jusqu'à 24 mille marcs d'argent fin, dans une année.

(27a) Salmon dit qu'on y a levé jusqu'à dix mille hommes de troupes.

On observe le fait général suivant: la formulation au passé composé tend à devenir aspectuellement ambiguë, et peut être interprétée aussi bien comme un énoncé au parfait existentiel que comme un énoncé au prétérit (i.e. désignant une occurrence spécifique et temporellement repérée de la situation). Il est intéressant d'analyser les exemples qui échappent à cette règle: (23a), (26a) et (27a).

Dans (23a), l'interprétation comme prétérit est exclue en raison de l'adverbial bien des fois, quantifieur de fréquence, qui sélectionne une lecture existentielle (voir propriété (vi)); dans (26a), l'interprétation comme prétérit est également exclue, en raison de l'expression jusqu'à 24 mille marcs d'argent fin et du complément dans une année, cette double indication supposant en effet plusieurs évaluations sur plusieurs années; dans (27a), enfin, l'interprétation comme prétérit est exclue également, en raison de l'expression jusqu'à dix mille hommes de troupes, qui implique qu'il y a eu plusieurs levées de troupes. Au total on constate donc que dans ces trois exemples,

13 La seule étude que nous ayons trouvée, qui établit un rapprochement entre les surcomposés et la valeur existentielle, est celle de Squartini (I998: 205), qui voit dans certains exemples de surcomposés 'rather an "experiential" flavour'. Carruthers (I998: I45) note quant à elle que les locuteurs qui emploient ce surcomposé emploient aussi le passé composé dans des contextes sémantiques analogues. 
il y a, de fait, une quantification de la fréquence, du moins une forme d'itérativité, et que c'est cette information qui induit quasi mécaniquement une interprétation existentielle du passé composé. Notons encore que dans (25a), l'interprétation comme prétérit, quoique possible, produirait cependant une sorte de contresens: après avoir écrit On a réalisé un beau score: $10 \propto u f s$, on voit mal qu'on puisse écrire, avec une valeur de prétérit: Il faut savoir qu'on est rentré avec un seul cuf.

On observe en outre que les formulations (24a) et (25a) perdraient leur ambiguité si on leur adjoignait un quantifieur de fréquence comme parfois. Elles ne pourraient alors être interprétées que comme des parfaits existentiels, c'est-à-dire comme des formulations pratiquement synonymes de (24) et (25):

(24b) Les habitants [...] racontent beaucoup d'histoires sur un vieux nain qu'on a parfois vu sur leurs pâturages.

(25b) On a réalisé un beau score: Io œufs. Il faut savoir qu'on est parfois rentré avec un seul oeuf.

Toutes ces manipulations indiquent que, pour être interprété comme parfait existentiel, le passé composé est fortement tributaire de ce que peut fournir le contexte comme indices interprétatifs. Si ces indices sont insuffisants, alors l'interprétation flotte entre deux, voire trois lectures (résultative, prétéritale, existentielle). En revanche, le surcomposé-E ne se trouve jamais dans ce type de dépendance. Il paraît bel et bien exprimer, comme forme grammaticale sui generis, la valeur de parfait existentiel.

On pourrait ajouter que, de même que le passé composé à valeur de prétérit suppose toujours une localisation temporelle 'définie' (comme le dit Michaelis (I998) à propos du simple past anglais), même quand celle-ci n'est pas explicitement donnée, de même le surcomposé-E suppose toujours une indication qu'on pourrait traduire par des expressions comme parfois à une certaine époque, parfois dans le passé, etc. Or, cette indication comporte deux éléments: 'à une certaine époque' ou 'dans le passé' désignent l'intervalle de validation nécessaire à la prédication existentielle; 'parfois' indique la quantification. La spécificité du surcomposé-E relativement au passé composé en emploi existentiel est précisément qu'il incorpore ces deux informations. C'est en cela qu'on est fondé à affirmer que le surcomposéE grammaticalise la valeur de parfait existentiel. L'exemple (27) est intéressant à cet égard, puisqu'on y trouve un surcomposé-E (sans adverbial) suivi d'un passé composé à valeur de parfait existentiel (modifié par l'adverbial autrefois). ${ }^{14}$

\section{L'intervalle de VAlidation}

Nous avons vu au début de cet article que l'intervalle de validation du passé composé à signification de parfait existentiel s'étend par défaut jusqu'au moment de la parole. Qu'en est-il du surcomposé-E?

${ }^{14}$ Clédat (I926: 44) notait qu'il y a une sorte d'équivalence entre il a eu fait et il a fait jadis. 
Notre hypothèse sur ce point est la suivante: l'intervalle de validation du surcomposé-E est, par défaut, entièrement localisé dans le passé, la borne terminale de cet intervalle (son terminus ad quem) étant elle-même située dans le passé. Il en résulte assez naturellement qu'un des rendements discursifs du surcomposé-E est l'expression d'un contraste entre le passé et le présent (ce qui est affirmé du passé étant, explicitement ou implicitement, nié du présent). C'est exactement ce qu'on observe dans les exemples (25)-(27): (25) compare la récolte d'œufs actuelle avec les récoltes passées; (26) compare la production actuelle d'une mine d'argent avec sa production antérieure; (27) compare la population d'une ville à deux époques différentes, en prenant comme étalon de comparaison le nombre d'homme de troupe qu'on pouvait y lever. Près de la moitié des exemples de surcomposés-E de notre corpus figurent dans un contexte de ce type (estimation faite sur un corpus de quelque cent items). Voici d'autres exemples de cette routine discursive consistant à mettre en contraste passé et présent:

(28) Malory est plus susceptible et une petite tape non intentionnelle finit en mélodrame alors que dans ses périples de casse cou elle s'est eu fait bien plus mal. (http://blog.aufeminin.com/)

(29) cette année la rencontre a lieu à Lausanne, mais ça s'est eu fait à Paris, ça s'est eu fait à Lyon, ça s'est eu fait à Montréal (locuteur lausannois, 2008)

(30) Oui, j'ai eu écrit des lettres d'amour à mon chéri et il aimait bien les recevoir. Maintenant je lui écrit des petits mots doux ou je lui envoie des mails lorsqu'il est au boulot. (http://forum.doctissimo.fr/)

(3 I) Je décortique les secondes, et plus elles passent, plus je me dis que je n'ai jamais vu passer le temps aussi vite! C'est vrai, alors que j'ai eu trouvé les minutes longues, voilà que maintenant les heures sont courtes! (http://ruptures.bleublog.lematin.ch/)

(32) - Loisir?

- ordinateur, internet et informatique... la faute à qui? la faute à Guppy..!!

J'ai en lu, j'ai en écrit, j'ai eu peint et dessiné, j'ai eu marché et visité, j'ai eu éconté et joué de la musique, j'ai eu regardé des films au cinoche, j'ai eu fait du tir à l'arc, j'ai eu fait du canevas et autres activités manuelles et extra-guppy. ...!!! Mais, tout excès à une fin... je me la souhaite pour bientôt..! (http://chezclo.free.fr/, 'Guppy' est le nom d'un portail web.)

L'exemple (3 I) est particulièrement intéressant. Il comporte successivement un passé composé à lecture existentielle (je n'ai jamais vu passer le temps aussi vite) et un surcomposé-E (alors que j'ai eu trouvé les minutes longues). Mais, tandis que l'intervalle de validation du passé composé s'étend jusqu'au présent de l'énonciation ('dans le passé et jusqu'à maintenant je n'ai jamais vu passer le temps aussi vite'), l'intervalle de validation du surcomposé-E s'étend jusqu'à une limite située elle-même dans le passé ('dans le passé et jusqu'à une date située dans le passé, il est arrivé que je trouve les minutes longues'). La localisation dans le passé du terminus ad quem de l'intervalle de 
validation paraît bien être ce qui fait la spécificité du surcomposé-E relativement à celle du passé composé ${ }^{15}$.

Nous avons en outre noté que cette mise en contraste entre passé et présent concerne assez souvent un paramètre gradable, sinon quantifiable (Apothéloz, à par.): par exemple le nombre d'œufs récoltés, pour (25). Ce phénomène peut également être observé dans (26)-(28) et dans (3 I).

Cette différence a des conséquences pragmatiques. Ainsi, les formulations au surcomposé-E déclenchent des inférences particulières concernant le présent de l'énonciation. Par exemple, les formes verbales de la longue énumération de (32) (j'ai eu lu, j'ai eu écrit, j'ai eu peint, etc.) suggèrent que maintenant je ne lis plus, maintenant je n'écris plus, et ainsi de suite. Une telle inférence, quoique possible, ne présenterait pas du tout la même certitude avec des passés composés.

Nous tenons peut-être là l'explication d'une description qui est devenue pratiquement un stéréotype sur le surcomposé-E: celle consistant à dire qu'il exprime l'idée d'un passé 'révolu' ou 'lointain' (e.g. Clédat, I926; Paesani, 200 I, cité par Schaden, 2007). Dans le même ordre d'idée, nous partageons les doutes formulés par Sthioul (2006) à propos des valeurs subjectives ou expressives si souvent attribuées au surcomposé-E (expression d'une nostalgie relativement aux faits évoqués, insistance sur la nature exceptionnelle de ces derniers, etc.). Selon nous, ces effets de sens ne sont que des conséquences indirectes de la conjonction de deux faits: la valeur de parfait existentiel, et la localisation dans le passé de l'intervalle de validation, y compris son terminus ad quem. Un troisième fait peut venir renforcer cet effet de sens: le surcomposé-E est propre au discours (et non au récit), et donc fortement corrélé à la Ière personne, ainsi que l'a noté Carruthers (I994).

Dans notre caractérisation initiale de l'intervalle de validation du passé composé employé comme parfait existentiel, nous avons indiqué qu'il s'étend par défaut jusqu'au moment de l'énonciation. L'indication 'par défaut' vise à rendre compte du fait que ce tiroir est sous-spécifié quant à l'extension de son intervalle de validation. En réalité, un passé composé employé comme parfait existentiel peut parfaitement s'accommoder d'un intervalle de validation situé complètement dans le passé. Mais le terminus ad quem de cet intervalle doit alors être explicitement signalé, ou être inférable à partir d'une autre information. Nous avons déjà rencontré ce phénomène avec des exemples comme (7) et (I 3). Entre les locuteurs 'possédant' et ne 'possédant' pas le surcomposé-E, il n'y a donc qu'une différence dans les moyens utilisés. Un passé composé employé comme parfait existentiel, modifié par un adverbial fermant à droite son intervalle de validation, équivaut à un surcomposé-E sans adverbial.

15 Notons que (32) présente une énumération non chronologique tout à fait caractéristique des emplois discursifs du parfait existentiel. 


\section{UN Pléonasme: Le PRÉdicat existentiel FLéChi AU SURCOMPOSÉ-E}

Nous terminerons ces analyses en signalant que le tour impersonnel il est arrivé que, en dépit du fait qu'il expose la valeur existentielle, est parfois lui-même fléchi au surcomposé-E. Il y a alors une sorte de pléonasme sur l'existentialité, celle-ci étant exprimée et par le surcomposé-E et par le verbe du tour impersonnel. Voici quelques attestations de ce phénomène:

(33) quand je ne suis pas content d'un tableau, je dors très mal la nuit. Cela m'est eu arrivé de me lever à deux heures du matin, parce que cela m'a fait tilt et j'ai trouvé le défaut et j'ai vite été corrigé. (http://www.treffpunktboulevard.ch/TV/)

(34) Il m'est eu arrivé de me plaindre de la stabilité de certaines applications. Quand on a un problème de stabilité de ce genre, il faut alors faire une mise à jour de l'application concernée. (http://boltıo24.over-blog.com/)

(35) Il m'est eu arrivé d'en acheter, quand ils sont en prix baissé. Les enfants les trouvent délicieux [...]. (http://www.bebe.ch/drupal/)

(36) comme ça m'est déjà eu arrivé, je viens de t'écrire un monstre long mail (http://vivineberline.blogspot.com/)

L'intérêt que présente la construction impersonnelle par rapport à l'autre formulation (par exemple, pour (33): je me suis eu levé à deux heures du matin) n'est pas seulement d'exposer la valeur existentielle. Elle permet également de formuler de façon syntaxiquement autonome la prédication existentielle et de signifier anaphoriquement la proposition qui est sous sa portée. C'est ce qui se passe dans des énoncés comme ça m'est arrivé ou ça m'est eu arrivé, dont un contenu propositionnel entier doit être récupéré dans le contexte:

(37) [il est question d'une panne informatique]

Ça m'est eu arrivé, peut-être que cela vient du navigateur (http://paranormal.forumpro.fr/)

Il est possible que ces emplois anaphoriques du tour impersonnel jouent un certain rôle dans la promotion de ce tour, même quand le tiroir choisi signifie par lui-même la valeur de parfait existentiel, comme c'est le cas du surcomposé-E. D'ailleurs, d'autres expressions peuvent venir accroître encore le pléonasme et, en quelque sorte, sur-signifier l'existentialité. C'est le cas de l'adverbe déjà dans (36). De sorte que dans cet exemple, l'existentialité est triplement marquée: par le sens même du verbe impersonnel, par le tiroir auquel ce dernier est fléchi (surcomposéE) et par l'adverbe déjà.

Des exemples comme ceux listés ci-dessus conduisent inévitablement à se demander s'il y a une différence sémantique entre (38) et (38'), (39) et (39'), (40) et (40'), etc.

(38) cela m'est eu arrivé de me lever à deux heures du matin...

$\left(38^{\prime}\right)$ je me suis eu levé à deux heures du matin. . .

(39) il m'est eu arrivé de me plaindre de... 
$\left(39^{\prime}\right)$ je me suis eu plaint de...

(40) il m'est eu arrivé d'en acheter...

$\left(40^{\prime}\right)$ j’en ai eu acheté...

Ces formulations (avec et sans le tour impersonnel) sont tout à fait régulières et communes chez les locuteurs qui 'possèdent' le surcomposé-E. Notre sentiment est qu'il n'y a aucune différence sémantique entre elles, mis à part le pléonasme. Cependant, il n'est pas impossible qu'en présence d'une construction passive, comme j'ai eu été réveillé, une certaine préférence soit accordée au tour impersonnel, qui permet d'éviter la succession de trois participes passés - d'où (4I):

(4I) Il m'est eu arrivé d'être réveillé assez tôt le matin par des personnes d'un autre fuseau horaire. (http://www.pauleau-maximilien.fr/)

\section{BILAN ET CONCLUSION}

$\mathrm{Au}$ terme de ces analyses, il nous parait maintenant bien établi que, pour les sujets parlants qui en possèdent la grammaire, la forme verbale qualifiée de 'passé surcomposé régional' (surcomposé-E) appartient à l'arsenal des moyens grammaticaux permettant de produire la valeur de parfait existentiel, et donc d'exprimer le concept d'existence. En comparaison avec le passé composé, le surcomposé-E présente les particularités suivantes.

En premier lieu, il témoigne d'une véritable grammaticalisation de la valeur existentielle. Pratiquement cela signifie que, contrairement au passé composé, il ne dépend pas du contexte pour produire cette valeur. Sur un plan théorique, cette idée se heurte cependant au fait que cette même forme grammaticale peut être observée avec une tout autre valeur aspectuelle, le plus souvent résultative, en particulier dans les subordonnées temporelles (quand il est utilisé pour corréler temporellement deux situations). Il conviendrait toutefois de vérifier si, chez les sujets parlants 'possédant' le surcomposé-E, le passé surcomposé en subordonnée temporelle existe bel et bien et fait partie de leurs pratiques grammaticales. Or selon l'enquête de Walter (I98I), il semble que dans la région franco-provençale, les formes surcomposées n'apparaissent pratiquement que sous la forme du surcomposé-E. Cette donnée ne peut que conforter la thèse de la grammaticalisation évoquée plus haut.

En second lieu, nous avons établi que le surcomposé-E et le passé composé à valeur de parfait existentiel different également dans la manière dont ils prédéfinissent leur intervalle de validation, i.e. l'intervalle temporel à l'intérieur duquel la prédication d'existence exerce son effet. Dans le cas du passé composé, cet intervalle s'étend par défaut jusqu'au moment de l'énonciation, qui en constitue le terminus ad quem. Cette propriété implique alors, comme plusieurs auteurs l'ont relevé, qu'il n'est pas exclu que la situation désignée puisse encore se reproduire. En revanche, dans le cas du surcomposé-E, le terminus ad quem de l'intervalle de validation est localisé par défaut dans le passé. Cette différence explique vraisemblablement pourquoi ce tiroir est si souvent utilisé dans des 
routines discursives où s'exprime un contraste temporel entre le passé et le présent. Elle explique donc également pourquoi le surcomposé-E, contrairement au passé composé existentiel, exclut en principe que la situation désignée puisse encore se reproduire.

Adresse de l'auteur:

Denis Apothéloz

Université de Nancy 2

U.F.R. Sciences du langage

23, bd Albert 1er

B.P. 33-97

54015 NANCY (France)

e-mail:denis.apotheloz@univ-nancy2.fr

\section{REFERENCES}

Apothéloz, D. (2009). La quasi-synonymie du passé composé et du passé surcomposé existentiels. Pratiques, I4I-I42: 98-I2O.

Apothéloz, D., Brandt, P.-Y., Quiroz, G. (1993). The function of negation in argumentation. Journal of Pragmatics, I9: 23-38.

Apothéloz, D., Nowakowska, M. (à par.). Note sur la résultativité et la valeur de parfait: leur expression en polonais. Cahiers Chronos.

Ayres-Bennett, W., Carruthers, J. (I992). 'Une regrettable et fort disgracieuse faute de français'?: The description and analysis of the French surcomposés from I 530 to the present day. Transactions of the Philological Society, 90.2: 219-257.

Barceló, G., Bres, J. (2006). Les temps de l'indicatif en français. Paris: Ophrys.

Beauzée, N. (I765). Article 'Tems'. In: Encyclopédie ou Dictionnaire raisonné des sciences, des arts et des métiers, vol. I6, pp. 96-I I7.

Benveniste, E. (1959). Les relations de temps dans le verbe français. Bulletin de la Société de Linguistique, 54.I. Repris dans Problèmes de linguistique générale, 1. Paris: Gallimard, I966, 237-250.

Brun, A. (I93 I). Le français de Marseille. Marseille: Institut Historique de Provence.

Carruthers, J. (I994). The passé surcomposé régional: towards a definition of its function in contemporary spoken French. Journal of French Language Studies, 4: I7I-I90.

Carruthers, J. (I998). Surcomposé 'general' et surcomposé 'régional': deux formes distinctes? Dans: G. Ruffino (dir.), Atti del XXI Congresso Internazionale di Linguistica e Filologia Romanza, Vol. 2. Tübingen: Max Niemeyer, pp. I43-I 54 .

Carruthers, J. (I999). A problem in sociolinguistic methodology: investigating a rare syntactic form. Journal of French Language Studies, 9: I-24.

Clédat, L. (I926). Les tens (sic) composés et surcomposés. Revue de philologie française et de littérature, 38: 33-47.

Cohen, D. (1989). L'aspect verbal. Paris: Presses Universitaires de France.

Comrie, B. (1976). Aspect. An Introduction to the Study of Verbal Aspect and Related Problems. Cambridge: Cambridge University Press.

Confais, J.-P. (I995). Temps, mode, aspect. Les approches des morphèmes verbaux et leurs problèmes à l'exemple du français et de l'allemand. Toulouse: Presses Universitaires du Mirail. 
Cornish, F. (2005). Une approche pragmatico-discursive des phrases 'thétiques'. Dans: F. Lambert et H. Nølke (dir), La syntaxe au coeur de la grammaire. Recueil offert en hommage pour le $60^{e}$ anniversaire de Claude Muller. Rennes: Presses Universitaires de Rennes, pp. $75-84$.

Cornu, M. (1953). Les formes surcomposées en français. Berne: A. Francke.

Dahl, Ö. (1985). Tense and Aspect Systems. Oxford: Blackwell.

Dauzat, A. (1954). A propos des temps surcomposés: surcomposé provençal et surcomposé français. Le français moderne, 22: 259-262.

Desclés, J.-P., Guentchéva, Z. (2003). Comment déterminer les significations du passé composé par une exploration contextuelle. Langue française, I 38: 48-60.

Engel, D. M. (I996). Le passé du passé. Word, 47.I: 4I-62.

Foulet, L. (I925). Le développement des temps surcomposés. Romania, 51: 203-252.

Frei, H. (1929). La grammaire des fautes. Bellegarde: Société anonyme des arts graphiques de France.

Géa, J.-M. (1995). Entre norme et usage: quelques formes surcomposées dans les lettres de deux soldats méridionaux de la Grande Guerre. Langage et Société, 7I: 65-85.

Gougenheim, G. (1939). Système grammatical de la langue française. Paris: d'Artrey.

Guentchéva, Z. (I990). Temps et aspect: l'exemple du bulgare contemporain. Paris: Editions du CNRS.

Guiraud, P. (1965). Le français populaire. Paris: PUF.

Hill, J. K. (I984). 'A la recherche de temps perdus': The double compound forms of the verb in present-day French. Word, 35. I: 89-II2.

Imbs, P. (1960). L'emploi des temps verbaux en français moderne. Paris: Klincksieck.

Jespersen, O. (1924). The Philosophy of Grammar. London: George Allen \& Unwin. Traduction française, La philosophie de la grammaire. Paris: Minuit, I97I.

Jolivet, R. (I984). L'acceptabilité des formes verbales surcomposées. Le français moderne, 3.4: I $59-\mathrm{I} 82$.

Jolivet, R. (I986). Le passé surcomposé: emploi “général” et emploi "régional”. Examen des insertions dans le syntagme verbal surcomposé. Dans: Mélanges d'onomastique linguistique et philologie offerts à M. Raymond Sindou, par ses collègues, ses amis et ses élèves, II, I09-II6.

Karolak, S. (2007). Składnia francuska o podstawach semantycznych, tom I. Kraków: Collegium Columbinum.

Karolak, S. (à par.). L'aspect dans une langue: le français. Travaux de linguistique.

Kęsik, M. (1998). Variations sur le thème de l'impersonnel. Lublin: Widawnictwo Uniwersytetu Marii Curie-Skłodowskiej.

Kiparsky, P. (s.d.). Event structure and the perfect. (adresse: www.stanford.edu/ $\sim$ kiparsky/Papers/semanticsfest.pdf)

Kleiber, G. (200I). Indéfinis: lecture existentielle et lecture partitive. Dans: G. Kleiber, B. Laca et L. Tasmowski (dir.), Typologie des groupes nominaux. Rennes: Presses Universitaires de Rennes, pp. 47-97.

Kuroda, S.-Y. (I973). Le jugement thétique et le jugement catégorique; exemples tirés de la syntaxe japonaise. Langage, 30 : 8 I-I IO.

Laca, B. (2005). Périphrases aspectuelles et temps grammatical dans les langues romanes. Dans: H. B. Z. Shyldkrot et N. Le Querler (dir), Les périphrases verbales. Amsterdam: J. Benjamins, pp. 47-66.

Lambrecht, K. (1994). Information Structure and Sentence Form. Topic, Focus, and the Mental Representations of Discourse Referents. Cambridge: Cambridge University Press. 
Leech, G. N. (I97I). Meaning and the English Verb. London: Longman.

Martinet, A., sous la dir. de (1979). Grammaire fonctionnelle du français. Paris: CrédifDidier.

McCawley, J. D. (I97I). Tense and time reference in English. Dans: C.J. Fillmore, D.T. Langendoen (dir.), Studies in Linguistic Semantics. New York: Holt, Rinehart and Winston, pp. 96-I I3.

McCawley, J. D. (I98I). Notes on the English perfect. Australian Journal of Linguistics, I: $8 \mathrm{I}-90$.

Michaelis, L. A. (1998). Aspectual Grammar and Past-time Reference. London, New York: Routledge.

Nilsson-Ehle, H. (1953). Remarques sur les formes surcomposées en français. Studia Neophilologica, 26. I-3: I 57-I67.

Paesani, K. A. (200I).Syntax and semantics of the passé surcomposé in modern French. Thèse de doctorat, Indiana University.

Reichenbach, H. (I947). Elements of Symbolic Logic. London, New York: Macmillan.

Ritz, M.-E. (2002). The semantics of the passé composé in contemporary French: towards an unified representation. Cahiers Chronos, 9: 3 I-50.

Schaden, G. (2007). La sémantique du parfait. Etude des 'temps composés' dans un choix de langues germaniques et romanes. Thèse de doctorat, Université Paris 8.

Squartini, M. (1998). Verbal Periphrases in Romance. Aspect, Actionality, and Grammaticalization. Berlin, New York: de Gruyter.

Stefanini, J. (1954). La tradition grammaticale française et les temps surcomposés. Annales de la Faculté des Lettres d'Aix, 28: 67-108. Réédité dans: Linguistique et langue française. Textes de J. Stefanini réunis par P. Bonnefois. Paris: Editions du CNRS., I992, pp. 37-74.

Sthioul, B. (2006). Le(s) passé(s) surcomposé(s): temps, aspect, subjectivité. Travaux neuchâtelois de linguistique, 45: I I 5-I 32 .

Tesnière, L. (1939). Théorie structurale des temps composés. Dans: Mélanges de linguistique offerts à Charles Bally. Genève: Georg, pp. I53-183.

Vet, C. (1980). Temps, aspects et adverbes de temps en français contemporain. Genève: Droz.

Vet, C. (I992). Le passé composé: contextes d'emploi et interprétation. Cahiers de Praxématique, I9: 37-59.

Walter, H. (I98I). Le surcomposé dans les usages actuels du français. Dans: Actants, voix et aspects verbaux. Angers: Presses de l'Université d'Angers, pp. 24-44.

Walter, H. (1988). Le français dans tous les sens. Paris: Laffont.

Wilmet, M. (I997). Grammaire critique du français. Louvain: Duculot. 